\title{
A self-dependency constraint in the simply typed lambda calculus
}

\author{
Aleksy Schubert* \\ Institute of Informatics \\ Warsaw University \\ ul. Banacha 2 \\ 02-097 Warsaw \\ Poland
}

\begin{abstract}
We consider terms of simply typed lambda calculus in which copy of a subterm may not be inserted to the argument of itself during the reduction. The terms form wide class which includes linear terms. We show that corresponding variant of the dual interpolation problem i.e. problem in which all expressions can be restricted to terms of this kind. Thus the model for this kind of expressions can admit fully abstract semantics.
\end{abstract}

Classification: semantics, logic in computer science.

\section{Introduction}

The higher-order matching problem (HOM) consists in solving certain equations in the simply typed $\lambda$-calculus. The equations have the form $M \doteq N$ where the unknowns occur only in the term $M$. HOM is a restricted version of the higherorder unification problem (HOU) which has several applications in the field of automated theorem proving. Unfortunately, the higher-order unification problem has been proved undecidable [Gol81]. Despite this sad result HOU has attracted a significant attention and is used as a tool in automated theorem proving (e.g. in Isabelle [Pau89]) and higher-order logic programming (e.g. in $\lambda$-Prolog [MN88]). As a special case of HOU the higher-order matching problem can be applied in various situations in these areas (see e.g. [Har96]).

The higher-order matching problem has also connections with semantics of the simply typed $\lambda$-calculus [Sta82]. It is strongly connected with the problem of $\lambda$-definability. The problem of $\lambda$-definability is to decide whether a given function from some function space over a finite base can be defined by a $\lambda$-term. The problem of $\lambda$-definability is undecidable which corresponds to the fact that there is no way to precisely describe a fully-abstract model for the simply typed $\lambda$ calculus [Loa01]. The higher-order matching problem defines a stronger notion of definability for which it is not known if it is decidable. Semantical investigations in simply typed lambda calculus have some practical impact as they are strongly connected to the area of program transformations (see e.g. [dMS01]).

\footnotetext{
* This work was partly supported by KBN grant 7 T11C 028 20; part of the work was conducted while the author was a winner of the fellowship of The Foundation for Polish Science (FNP).
} 
The higher-order matching problem has a long history. The problem was posed by G. Huet in his $\mathrm{PhD}$ thesis [Hue76]. It was considered in some older papers ([Wol89,Bax76]), but it gained more attention in the last years. V. Padovani presented a new approach to higher-order matching. He proved that higher-order matching is decidable without restriction on the order but with a restriction on the term $N$ - it must be a single constant of a base type [Pad95a]. In addition, R. Loader presented [Loa] the undecidability of unrestricted higher-order matching, but for $\beta$-equality (the standard formulation requires $\beta \eta$-equality). This was supplemented by results that take into account restrictions on domains of solutions [dG00,DW02,SSS02].

This paper expands significantly the technique used in [Pad95a] and proposes a new interesting class of terms. Informally, a term $M$ is self-independent when in each self-independent context and for each subterm $N$ of the term if the evaluation of the subterm $N$ is executed somewhere in the reduction then all its arguments may not contain a copy of $N$. For example the term

$$
\lambda x:(\alpha \rightarrow \alpha) \rightarrow \alpha \rightarrow \alpha . x\left(\lambda z_{1} . f\left(z_{1}, z_{1}\right)\right) a
$$

is self-independent since the only self-independent terms that can be substituted for $x$ are the identity, and the constant term and these can only result in forgetting of most of the term of in copying $a$ to $z_{1}$. Accordingly the term

$$
\lambda x:((\alpha \rightarrow \alpha) \rightarrow \alpha \rightarrow \alpha) \rightarrow(\alpha \rightarrow \alpha) \rightarrow \alpha . x\left(\lambda z_{1} . z_{1}\left(z_{1}(a)\right) f\right.
$$

is not self-independent, as in one if possible contexts (when the identity is substituted for $x$ ) the term $f$ occurs in the argument of the first $z_{1}$. This class extends the class of linear terms. Moreover, presented proof gives a nice syntactical characterisation of this class. We present a proof of decidability for the dual interpolation problem with an additional restriction on solutions and instances - they must be self-independent terms. This result extends to higher-order matching for this kind of expressions.

\section{Preliminaries}

Assume we are given a set $\mathcal{B}$ of base types. Let $\mathcal{T}_{\mathcal{B}}$ be the set of all simple types over $\mathcal{B}$ defined as the smallest set containing $\mathcal{B}$ and closed on $\rightarrow$. We shall often omit the subscript $\mathcal{B}$ if $\mathcal{B}$ is clear from the context or unimportant.

In this paper we limit ourselves to the case where $\mathcal{B}$ is a singleton $\{\iota\}$.

The set of simply typed terms is defined based on the set of pre-terms. The set $\lambda_{\rightarrow}^{*}$ of simply typed pre-terms contains an infinite, countable set of variables $V$, a countable set of constants $C$, and a map $T: V \cup C \rightarrow \mathcal{T}_{\mathcal{B}}$ that indicates types of symbols. We assume that there exists an infinite number of variables of a type $\alpha$. This set is also closed on the application and $\lambda$-abstraction operations. We will usually write $s: \tau$ to denote the fact that a pre-term $s$ has a type $\tau$. As usual, we deal with $\alpha$-equivalence. The symbol $[s]_{\alpha}$ denotes the $\alpha$-equivalence class of $s$. The set $\lambda_{\rightarrow}$ is defined as a quotient of $\lambda_{\rightarrow}^{*}$ by $\alpha$-equivalence. The elements of $\lambda_{\rightarrow}$ are denoted usually by $M, M_{1}, \ldots, N, N_{1} \ldots$ etc. as well as the 
elements of $\lambda_{\rightarrow}^{*}$ are denoted by $s, s_{1}, \ldots, t, t_{1}, \ldots$ etc. The notion of a closed term is defined as usual, similarly the set $\mathrm{FV}(M)$ of free variables in $M$. The symbol Const $_{M}$ denotes the set of constants occurring in $M$.

We denote by $T(\tau, C)$ the set of all closed terms of type $\tau$ built-up of constants from the set $C$. The notion of sorted set is of some usefulness here. The family of sets $T^{C}=\{T(\tau, C)\}_{\tau \in \mathcal{T}_{\mathcal{B}}}$ is an example of a sorted set. We adopt standard $\in$ notation to sorted sets.

The term Const $_{M}$ denotes the set of constants that occur in $M$. This notation is extended to sets (and other structures) of terms.

The order of a type $\tau$, denoted by $\operatorname{ord}(\tau)$ is defined inductively as

$-\operatorname{ord}(\iota)=0$ for $\iota \in \mathcal{B}$

$-\operatorname{ord}\left(\tau_{1} \rightarrow \tau_{2}\right)=\max \left(\operatorname{ord}\left(\tau_{1}\right)+1, \operatorname{ord}\left(\tau_{2}\right)\right)$.

The notion of order extends to terms and pre-terms. We define ord $(\mathrm{M})=$ $\operatorname{ord}(\tau) \operatorname{or} \operatorname{ord}(\mathrm{t})=\operatorname{ord}(\tau)$, where $\tau$ is the type of $M$ or $t$ respectively.

The notion of $\beta$-reduction $\left(\rightarrow_{\beta}^{*}\right)$ is defined as a congruent extension of the relation $(\lambda x . M) N \rightarrow_{\beta} M[x:=N]$ where $M[x:=N]$ denotes substitution of $N$ for $x$ with usual renaming of bounded identifiers. We sometimes write substitutions in the prefix mode as in $S(N)$.

The notion of $\beta \eta$-reduction $\left(\rightarrow_{\beta \eta}^{*}\right)$ is defined as a congruent extension of the above-mentioned $\rightarrow_{\beta}$ relation supplied with an additional rule $\lambda x . M x \rightarrow_{\eta} M$ where $M$ has no occurrence of $x$. In this paper, we deal with terms in $\beta$-normal, $\eta$-long form. The $\beta$-normal, $\eta$-long form for a term $M$ is denoted by $\operatorname{NFL}(M)$.

We call an instance of the higher-order matching problem each pair of simply typed $\lambda$-terms $\langle M, N\rangle$. We usually denote them as $M \doteq N$.

$A$ solution of such an instance is a substitution $S$ such that $S(M)={ }_{\beta \eta} N$.

We often restrict ourselves to the case when $N$ has no free variables. This restriction is not essential.

Definition 1 (the higher-order matching problem)

The higher-order matching problem is a decision problem - given an instance $M \doteq N$ of the higher-order matching problem whether there exists a solution of $M \doteq N$.

An interpolation equation is a pair of terms, usually written $x N_{1} \cdots N_{k} \doteq N$, such that $x$ is the only free variable in the left-hand side of the equation.

Let $\left\langle E, E^{\prime}\right\rangle$ of interpolation equations such that there exists a variable $x$ which occurs free in the left-hand side of each equation in $E \cup E^{\prime}$. We call $\left\langle E, E^{\prime}\right\rangle$ an instance of the dual interpolation problem. We also call $\left\langle E, E^{\prime}\right\rangle$ a dual set.

$A$ solution of such an instance is a term $P$ such that for each equation $\left[x N_{1} \cdots N_{k} \doteq N\right] \in E$ we have $P N_{1} \cdots N_{k}={ }_{\beta \eta} N$, and for each equation $\left[x N_{1} \cdots N_{k} \doteq N\right] \in E^{\prime}$ we have $P N_{1} \cdots N_{k} \neq \beta \eta N$.

Definition 2 (the dual interpolation problem)

The dual interpolation problem is a decision problem - given an instance $\left\langle E, E^{\prime}\right\rangle$ of the dual interpolation problem whether there exists a solution of $\left\langle E, E^{\prime}\right\rangle$.

The dual interpolation problem and the higher-order matching problem are connected in the following way: 
Theorem 1. The problem of higher-order matching reduces to the dual interpolation problem.

Proof. See [Pad95b,Pad96] or [Sch01].

\subsection{Self-independent terms}

We define self-independent terms by means of a marked reduction:

$$
\begin{aligned}
& (\lambda x . M) N \rightarrow_{\beta \#} M[x:=N] \\
& (M)^{\#} N \rightarrow_{\beta \#} M N .
\end{aligned}
$$

where the substitution $[x:=N]$ is defined so that $(P)^{\#}[x:=N]=(P[x:=N])^{\#}$.

A self-dependency is a term of the form $(M)^{\#} N_{1} \cdots N_{k}$ where either $M$ or one of $N_{i}$ contains the mark \#. For each term $M$ we define the set $\mathbb{M}^{\#}(M)$ as

$$
\mathrm{M}^{\#}(M)=\left\{C\left[x^{\#} N_{1} \cdots N_{k}\right] \mid M=C\left[x N_{1} \cdots N_{k}\right]\right\}
$$

$(C[\cdot]$ denotes a term with a single hole usually called context, substitution for $[\cdot]$ is not capture avoiding.)

All terms of a base type are self-independent. We say that a term $M$ is self-independent if for each sequence $N_{1}, \ldots, N_{n}$ of self-independent terms the outmost-leftmost reduction of each term $M^{\prime} N_{1} \cdots N_{n}: \iota$ where $\iota$ is a base type and $M^{\prime} \in \mathrm{M}^{\#}(M)$ does not include a term with a subterm being a selfdependency.

\section{Tools and definitions}

\subsection{Addresses in terms}

Let $t$ be a pre-term. We say that a sequence $\gamma$ of natural numbers is an address in $t$ iff

$-\gamma=\varepsilon$ (the empty sequence) or

$-\gamma=i \cdot \gamma^{\prime}$ where $t=\lambda x_{1} \ldots x_{m} \cdot u_{0} u_{1} \cdots u_{n}$ together with $0 \leq i \leq n$ and $\gamma^{\prime}$ is an address in $u_{i}$.

The set of all addresses in $t$ is written as $\operatorname{Addr}(t)$. The prefix order on addresses is written as $\gamma \preceq \gamma^{\prime}$. The strict version is denoted as $\gamma \prec \gamma^{\prime}$.

We say that a subterm $u$ is pointed out by an address $\gamma$ in $t$ iff

$-u=t^{\prime}$ where $t=\lambda x_{1} \ldots x_{m} \cdot t^{\prime}$, the term $t^{\prime}$ does not begin with $\lambda$ and $\gamma=\varepsilon$,

- or $u=t^{\prime}$ where $t=\lambda x_{1} \ldots x_{m} \cdot u_{0} u_{1} \cdots u_{n}$, and $\gamma=i \cdot \gamma^{\prime}$ together with $0 \leq i \leq n$ and $\gamma^{\prime}\left(u_{i}\right)=t^{\prime}$.

We write $\gamma(t)$ to denote $u$.

A graft of a term $u$ in $t$ at an address $\gamma$ is a term $t^{\prime}$ defined as

$-u$ iff $\gamma=\varepsilon$,

$-\lambda x_{1} \ldots x_{m} \cdot u_{0} u_{1} \cdots u_{i}^{\prime} \cdots u_{n}$ iff $\gamma=i \cdot \gamma^{\prime}$ and $t=\lambda x_{1} \ldots x_{n} \cdot u_{0} u_{1} \cdots u_{n}$ and $u_{i}^{\prime}$ is a graft of $u$ in $u_{i}$ at $\gamma^{\prime}$. 
We denote $t^{\prime}$ as $t[\gamma \leftarrow u]$. We sometimes use the notation $t[c \leftarrow u]$ where $c$ is a constant. This means that the term $u$ is grafted on every occurrence of the constant $c$.

Let $C$ be a set of fresh constants. We say that a pre-term $u$ is a $C$-pruning of a pre-term $t$ iff

$-u=t$,

- or $u=u^{\prime}[\gamma \leftarrow c]$ where $c \in C$ and $u^{\prime}$ is a $C$-pruning of $t$.

Let $t^{\prime}$ and $t^{\prime \prime}$ be $C$-prunings of a term $t$. We say that $t^{\prime} \preceq t^{\prime \prime}$ iff $\operatorname{Addr}\left(t^{\prime}\right) \subseteq$ $\operatorname{Addr}\left(t^{\prime \prime}\right)$. This pre-order may be viewed as an extension of the prefix order on sequences. Thus, we denote this relation by the same symbol. The strict version of the order is denoted by $\prec$. The above-mentioned notions easily extend to $\lambda$-terms, sets of $\lambda$-terms and sets of pre-terms.

\subsection{Matrix notation}

Every finite sequence of terms of the same type is called a column. We denote columns by symbols like $V, W$ etc. The symbol $V^{i}$ denotes the $i$-th element of the column $V$. The height of a column $V$ is the length of the sequence $V$. It is denoted by $|V|$. As all the elements of a column $V$ have the same type, we may use the notion of a type of the column $V$. When $V$ has a type $\tau$ then we denote this fact as $V: \tau$. We say that $V$ is a constant column when all its elements are the same.

Every finite sequence of terms is called a row. We denote rows by symbols like $R, Q$ etc. The symbol $R_{i}$ denotes the $i$-th element of the row $R$. The width of a row $R$ is the length of the sequence $R$ and is denoted as $|R|$. The operation of concatenation of columns $W, W^{\prime}$ or rows $R, R^{\prime}$ is denoted as $W W^{\prime}$ or $R R^{\prime}$.

Every finite sequence of columns is called a matrix. We denote matrices by symbols like $\overline{\mathrm{M}}, \overline{\mathrm{N}}$.

The symbol $\overline{\mathrm{M}}_{i}$ denotes the $i$-th column of $\overline{\mathrm{M}}$. The symbol $\overline{\mathrm{M}}_{i}^{j}$ denotes the $j$-th element of the $i$-th column of $\overline{\mathrm{M}}$. Similarly, $\overline{\mathrm{M}}^{i}$ denotes a row defined as $\left(\overline{\mathrm{M}}^{i}\right)_{j}=\overline{\mathrm{M}}_{j}^{i}$.

Let $\overline{\mathrm{M}}$ be a matrix and $W$ a column of a base type. We define a set of equations with the matrix $\overline{\mathrm{M}}$ and results $W$ as

$$
x \overline{\mathrm{M}}_{1}^{1} \cdots \overline{\mathrm{M}}_{n}^{1} \doteq W^{1}, \quad \cdots, \quad x \overline{\mathrm{M}}_{1}^{m} \cdots \overline{\mathrm{M}}_{n}^{m} \doteq W^{m} .
$$

We denote the set of equations as $[x \overline{\mathrm{M}} \doteq W]$. We extend the notation $\operatorname{NFL}(M)$ to vectors, rows and matrices: $\operatorname{NFL}\left(V M_{1} \cdots M_{n}\right), \operatorname{NFL}(M R)$ and $\operatorname{NFL}(M \bar{M})$. The notation Const $_{M}$ extends to matrices and vectors and means the set of constants occurring in the supplied matrix or vector.

\subsection{Approximations}

We say that a pair $W, W^{\prime}$ of columns is constant iff $W$ is constant as a column and $W^{\prime i} \neq W^{1}$ for all $i$.

An approximation of a pair of columns $W_{1}, W_{2}$ in a dual set $\mathcal{E}=\left\langle\left[x \overline{\mathrm{M}}_{1} \doteq\right.\right.$ $\left.\left.W_{1}\right],\left[x \overline{\mathrm{M}}_{2} \doteq W_{2}\right]\right\rangle$ of equations for the solution $M$ is any pair of columns $\widetilde{W}_{1}, \widetilde{W_{2}}$ of the heights $\left|W_{1}\right|,\left|W_{2}\right|$ such that 
- there exists an $\{a, b, 0, \ldots, l\}$-pruning $M^{\prime}$ of $M$ such that for each equation $\left[x \overline{\mathrm{M}}_{i, 1}^{k} \cdots \overline{\mathrm{M}}_{i, r}^{k} \doteq W_{i}^{k}\right] \in \mathcal{E}$ we have $\operatorname{NFL}\left(M^{\prime} \overline{\mathrm{M}}_{i, 1}^{k} \cdots \overline{\mathrm{Mi}}_{i, r}^{k}\right)=\widetilde{W_{i}^{k}}$,

- for $c \in\{a, b\}$ if $c$ occurs in $\widetilde{W_{j}^{i}}$ then $\widetilde{W}_{j}^{i}=c$,

- for each constant $c \in \mathbb{N}$ if some $\widetilde{W}_{j}^{i}=c$ then there exists $\gamma \succ \varepsilon$ and $k, l$ such that $\gamma\left(\widetilde{W_{l}^{k}}\right)=c$ (we say $c$ is guarded).

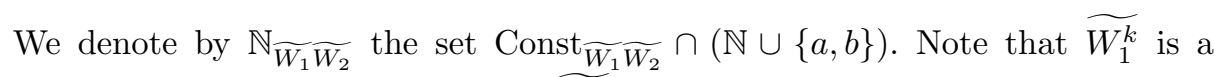
$\{a, b, 0, \ldots, l\}$-pruning of $W_{1}^{k}$ while $\widetilde{W_{2}^{k}}$ may not be a $\{a, b, 0, \ldots, l\}$-pruning of $W_{2}^{k}$.

For the sake of notational convenience we assume that for each approximation, the set $\mathbb{N}_{\widetilde{W} \widetilde{W^{\prime}}} \cap \mathbb{N}$ is an initial connected subset of $\mathbb{N}$, e.g. $\{0,1,2\}$ or $\{0,1\}$.

Let $\widetilde{W_{1}}, \widetilde{W_{1}^{\prime}}$ and $\widetilde{W_{2}}, \widetilde{W_{2}^{\prime}}$ be approximations of a pair of columns $W, W^{\prime}$. We write $\widetilde{W_{1}}, \widetilde{W_{1}^{\prime}} \preceq \widetilde{W_{2}}, \widetilde{W_{2}^{\prime}}$ when for each $k$ we have $\widetilde{W_{1}^{k}} \preceq \widetilde{W_{2}^{k}}$ and $\widetilde{W_{2}^{\prime k}} \preceq \widetilde{W_{2}^{\prime k}}$. It is easily verified that $\unlhd$ is a pre-order. The strict version of the order is denoted as $\triangleleft$.

We say that for an approximation $\widetilde{W}, \widetilde{W^{\prime}}$ of $W, W^{\prime}$ the term ${\widetilde{W^{\prime}}}^{k}$ meets $W^{\prime k}$ iff for each $k$ there exist $s_{a}, s_{b}, s_{0}, \ldots s_{l}$ such that

$$
W^{\prime k}={\widetilde{W^{\prime}}}^{k}\left[a \leftarrow s_{a}, b \leftarrow s_{b}, 0 \leftarrow s_{0}, \ldots, l \leftarrow, s_{l}\right] .
$$

A pair of columns $W, W^{\prime}$ is trivial iff for each $i, j$ we have $W^{i}=W^{\prime j}=c$, where $c$ is either $a$ or $b$.

Let $\left\langle[x \overline{\mathrm{M}} \doteq W],\left[x \overline{\mathrm{M}^{\prime}} \doteq W^{\prime}\right]\right\rangle$ be a dual set and $M$ its solution. We say that $\widetilde{W}, \widetilde{W^{\prime}}$ is a minimal approximation of $W, W^{\prime}$ iff there is no non-trivial $\widetilde{W_{1}} \widetilde{W_{1}^{\prime}} \triangleleft$ $\widetilde{W}, \widetilde{W^{\prime}}$.

We say that a term $M^{\prime}$ is a minimal pruning for minimal approximation $\widetilde{W}, \widetilde{W^{\prime}}$ iff $\widetilde{W}, \widetilde{W^{\prime}}$ is a minimal approximation of $W, W^{\prime}$ and there is no $M^{\prime \prime} \prec M^{\prime}$ such that $\operatorname{NFL}\left(M^{\prime \prime} \overline{\mathrm{M}}\right)=\widetilde{W}$ and $\operatorname{NFL}\left(M^{\prime \prime} \overline{\mathrm{M}^{\prime}}\right)=\widetilde{W^{\prime}}$.

Let $W, W^{\prime}$ be a pair of columns with a type $\tau_{1} \rightarrow \cdots \rightarrow \tau_{n} \rightarrow \iota$. We say that a pair of columns $V, V^{\prime}$ is a splitting pair of columns for $W, W^{\prime}$ iff there exist terms $M_{1}, \ldots, M_{n}$ such that the pair of columns $\operatorname{NFL}\left(V M_{1} \cdots M_{n}\right)$, $\operatorname{NFL}\left(V^{\prime} M_{1} \cdots M_{n}\right)$ is an approximation of $W, W^{\prime}$.

\subsection{Accessibility}

Let $E=[x \overline{\mathrm{M}} \doteq W]$ be a set of interpolation equations. We say that an address $\gamma$ is accessible in a term $M$ wrt. $E$ iff

$-\gamma$ is an address in $M$,

- there is an equation $\left[x \overline{\mathrm{M}}_{1}^{i} \cdots \overline{\mathrm{M}}_{n}^{i} \doteq W^{i}\right] \in E$ such that

$$
\mathrm{NF}\left(M[\gamma \leftarrow c] \overline{\mathrm{M}}_{1}^{i} \cdots \overline{\mathrm{M}}_{n}^{i}\right)
$$

has an occurrence of $c$, where $c$ is a fresh constant of a base type.

We say that an address is totally accessible iff for each equation in $E$ the condition (1) holds. 
We say that an address is totally head accessible iff for each equation in $E$ we have $\operatorname{NF}\left(M[\gamma \leftarrow c] \overline{\mathrm{M}}_{1}^{i} \cdots \overline{\mathrm{M}}_{n}^{i}\right)=c$, where $c$ is a fresh constant. Without loss of generality we may assume that $c$ is a constant of the base type.

Let $\left\langle E, E^{\prime}\right\rangle$ be a dual set and $M$ its solution. We say that an occurrence $\gamma$ is totally head accessible wrt. the dual set iff it is totally head accessible wrt. $E$ and $E^{\prime}$.

\subsection{Observational equivalence}

We introduce a notion of observational equivalence. This notion is very closely related to the dual interpolation problem. Roughly speaking, solutions of an instance of the dual interpolation problem form an equivalence class in this relation.

Let $\mathcal{R}=\left\{\mathcal{R}_{\tau}\right\}_{\tau \in \mathcal{T}}$ be an indexed family of sets containing $\lambda$-terms, and satisfying conditions

1. all terms in $\mathcal{R}$ are in $\beta$-normal, $\eta$-long form,

2. for each term $M \in \mathcal{R}$, there exists an $\alpha$-representant $s_{M}$ of $M$ such that for each subterm $t$ of the pre-term $s_{M}$ we have $[t]_{\alpha} \in \mathcal{R}$.

Such a set is called an observable.

The notion of an observable gives rise to a variation of the dual interpolation problem and the higher-order matching problem.

An instance of the dual interpolation problem for an observable $\mathcal{R}$ is a pair of sets $\mathcal{E}=\left\langle E, E^{\prime}\right\rangle$ of interpolation equations such that there exists a variable $x$ which occurs free in the left-hand side of each equation in $E \cup E^{\prime}$ and all right-hand sides of $E \cup E^{\prime}$ belong to $\mathcal{R}$.

The dual interpolation problem for an observable $\mathcal{R}$ is to decide whether an instance of the dual interpolation problem for the observable $\mathcal{R}$ has a solution.

Definition 3 (the higher-order matching problem for an observable)

An instance of the higher-order matching problem for an observable $\mathcal{R}$ is a pair of simply typed $\lambda$-terms $\langle M, N\rangle$ where $N \in \mathcal{R}$.

The higher-order matching problem for an observable $\mathcal{R}$ is to decide whether a given instance of the higher-order matching problem for the observable $\mathcal{R}$ has a solution.

The notion of an observable is also a base for a pre-order and an equivalence relation which, in turn, allow us to define a semantic structure for the simply typed $\lambda$-calculus.

Definition 4 (observational pre-order)

For each observable $\mathcal{R}$ we define an observational pre-order with respect to $\mathcal{R}$ as the relation $\sqsubseteq_{\mathcal{R}}$ on $\lambda$-terms such that

$M \sqsubseteq_{\mathcal{R}} M^{\prime}$ iff $M$ and $M^{\prime}$ have both the type $\sigma \rightarrow \tau$, and for each sequence of terms $N_{1}, \ldots, N_{n}$ with appropriate types and $n \geq 1$ we have that if $\operatorname{NFL}\left(M N_{1} \cdots N_{n}\right) \in \mathcal{R}$ then $M N_{1} \cdots N_{n}={ }_{\beta \eta}$ $M^{\prime} N_{1} \cdots N_{n}$ or

$M$ and $M^{\prime}$ are both of a base type and if $\operatorname{NFL}(M) \in \mathcal{R}$ then $M={ }_{\beta \eta} M^{\prime}$ 
Definition 5 (observational equivalence)

For each observable $\mathcal{R}$ we define an observational equivalence with respect to this observable as the relation on closed terms $\approx_{\mathcal{R}}$ such that

$$
M \approx_{\mathcal{R}} M^{\prime} \text { iff } M \sqsubseteq_{\mathcal{R}} M^{\prime} \text { and } M^{\prime} \sqsubseteq_{\mathcal{R}} M
$$

More details concerning this equivalence are in [Sch01]. In particular, we have the following theorem

Theorem 2. For each solvable instance $\mathcal{E}$ of dual interpolation for an observable $\mathcal{R}$ there exists an equivalence class of $\approx_{\mathcal{R}}$ such that all its elements are solutions of $\mathcal{E}$.

For each class $A$ of the relation $\approx_{\mathcal{R}}$ there exists an instance $\mathcal{E}$ of the dual interpolation problem for the observable $\mathcal{R}$ such that terms from $A$ solve $\mathcal{E}$.

There is an algorithm which for a type $\tau=\tau_{1} \rightarrow \cdots \tau_{n} \rightarrow \iota$ and complete sets of representants of $\approx_{\mathcal{R}}$ classes for types $\tau_{1}, \ldots, \tau_{n}$ generates a set $\mathcal{C}$ of instances such that for each $\approx_{\mathcal{R}}$ class $A$ in the type $\tau$ there is an instance $\mathcal{E}_{A}$ such that terms of $A$ are the only solutions of $\mathcal{E}_{A}$.

Proof. Easy conclusion from [Sch01].

\subsection{Transferring terms}

The proof in this paper is based on the following schema: We define transferring terms. We show that each solvable dual interpolation instance has a solution of this form. As the form is quite simple, we are able to enumerate the terms and this gives a proof that the dual interpolation problem is decidable.

This section contains the crucial definition of transferring terms. The definition is a little bit tedious, but we shall explain some of its elements later on in this section.

Definition 6 (transferring terms)

Let $n, m \in \mathbb{N}$ and let $C$ be a set of constants. A closed term $M: \tau_{1} \rightarrow \cdots \rightarrow$ $\tau_{p} \rightarrow \iota$ is an $(n, m, C)$-transferring term iff

1. $M=\lambda y_{1} \ldots y_{p} \cdot M^{\prime}$ and $M^{\prime}$ is a term over $C$ without any occurrence of $y_{i}$ where $i=1, \ldots, p$, or

2. $M=\lambda y_{1} \ldots y_{p} . f M_{1} \cdots M_{k}$, where

- $f \in C$ has a type $\sigma_{1} \rightarrow \cdots \rightarrow \sigma_{k} \rightarrow \iota$, and

- $M_{i}=\lambda z_{1} \ldots z_{r} . M_{i}^{\prime}$ where $M_{i}^{\prime}$ does not begin with $\lambda$, and

- $\lambda y_{1} \ldots y_{p} . M_{i}^{\prime}$ are $\left(n_{i}, m, C_{i}\right)$-transferring terms, and

$-n_{i}<n$, and

- $C_{i}=C \cup\left\{z_{1}, \ldots, z_{r}\right\}$, or

3. $M=\lambda y_{1} \ldots y_{p} \cdot y_{i} M_{1} \cdots M_{k}\left[a \leftarrow N_{a} \boldsymbol{y}, b \leftarrow N_{b} \boldsymbol{y}, 0 \leftarrow N_{0} \boldsymbol{y} \boldsymbol{x}_{\mathbf{0}}, \ldots, l \leftarrow N_{l} \boldsymbol{y} \boldsymbol{x}_{\boldsymbol{l}}\right]$, where

$-\boldsymbol{y}=y_{1}, \ldots, y_{p}$

- each $M_{i}$ is a closed term over $C \cup\{a, b, 0, \ldots, l\}$;

- each constant $0, \ldots, l$ occurs only once in $y_{i} M_{1} \cdots M_{k}$;

- $N_{a}$ and $N_{b}$ are $\left(n_{a}, m_{a}, C\right)$-transferring and $\left(n_{b}, m_{b}, C\right)$-transferring respectively; 


$$
\begin{aligned}
& -N_{j} \text { is }\left(n_{j}, m_{j}, C\right) \text {-transferring for each } j ; \\
& -n_{a}, n_{b} \leq n ; \\
& -m_{a}+m_{b} \leq m \text { and } m_{a}, m_{b} \neq m ; \\
& -n_{j}<n \text { and } m_{j} \leq n .
\end{aligned}
$$

Sometimes when $(n, m, C)$ are unimportant or clearly seen from the context, we shorten the name to transferring.

Suppose that case (3) is abridged so that there are no constants from $\mathbb{N}$. The resulting term $M$ may be presented as

$$
M=\lambda \boldsymbol{y} . C_{y_{k}}\left[y_{k} M_{1} \cdots\left(\lambda \boldsymbol{z} z_{j} \boldsymbol{z}^{\prime} \cdot M_{i}\right) \cdots M_{r}\right]
$$

Consider $\lambda \boldsymbol{z} z_{j} \boldsymbol{z}^{\prime} . M_{i}$. In this term, none of occurrences of $z_{j}$ in $M_{i}$ is in a subterm beginning with $y_{l}$ from $\boldsymbol{y}$.

When this constraint is applied, the number of occurrences of variables from $\boldsymbol{y}$ in an $(n, m, C)$-transferring term $M$ is bounded by $m$.

This simple picture is contaminated by the presence of constants from $\mathbb{N}$. We allow these constants to occur in subterms of $M$ beginning with variables $y_{l}$ from $\boldsymbol{y}$. This adds some flexibility and, consequently, expressive power to our terms. This flexibility is restricted, though. We have to pay for blocks that declare these variables with coins kept in $n$.

We sometimes treat informally this particular form of $\lambda$-terms as a syntactic form. In this syntactic notation, all substitutions from case (3) are actually present in a term. Formal development of this approach would involve a formalism similar to explicit substitutions formalisms.

\section{Transferring terms and dual interpolation}

Here is a theorem that relates the dual interpolation problem to transferring representatives. This is the central theorem of the paper.

Theorem 3. Let $\mathcal{E}$ be an instance of the dual interpolation problem. If $M$ is a solution of $\mathcal{E}$ then there exist $n, m \in \mathbb{N}$ together with a set of constants $C$ and an $(n, m, C)$-transferring term $M^{\prime}$ such that $M^{\prime}$ is a solution of $\mathcal{E}$, too.

Moreover, $n, m, C$ depend recursively on $\mathcal{E}$.

In order to prove this theorem we need two lemmas that enable some cleaning of sometimes excessively complicated terms.

\subsection{Skipping unimportant variables}

The induction step in the proof of Theorem 3 consists in splitting a dual set and finding transferring solutions for the results of the split. These solutions are based on a term $M$ that solves the whole set at the very beginning. This term is too complicated to be a compact solution of the split sets. During the process of construction of transferring solutions for them, we have to compact $M$ in several different ways. This section is devoted to the major step of the compactification. 
Below, we use $C$-prunings for $C=\{a, b\} \cup \mathbb{N}$. Thus we shorten the name $C$-pruning to pruning here. We impose a constraint on the shape of prunings performed on solutions. Constants from $\mathbb{N}$ may occur only once.

First of all, we have to determine what we want to skip.

Definition 7 (an unimportant occurrence)

Let $M=\lambda \boldsymbol{y} \cdot y_{i} M_{1} \cdots M_{k}$ be a term that solves a dual set $\mathcal{E}=\langle[x \overline{\mathrm{M}} \doteq W],[x \overline{\mathrm{M}} \doteq$ $\left.\left.W^{\prime}\right]\right\rangle$, and let $M^{\prime}$ be a pruning of $M$ that gives a minimal approximation of $W, W^{\prime}$. An occurrence $\gamma \cdot 0$ of a variable $z$ in $M^{\prime}$ is unimportant iff

$-\gamma$ is totally head accessible, and

- there is $\gamma^{\prime} \neq 0$ such that $\gamma \cdot \gamma^{\prime}$ is an occurrence of a variable bound on $\gamma$ and $\gamma \cdot \gamma^{\prime}$ is totally head accessible.

Note that unimportant occurrence has always the shape $\gamma \cdot 0$. We say that an unimportant occurrence $\gamma \cdot 0$ is maximal if there is no unimportant occurrence $\gamma \cdot \gamma^{\prime} \cdot 0$ where $\gamma^{\prime} \succ \varepsilon$.

Lemma 1. Let $M=\lambda \boldsymbol{y} . z M_{1} \cdots M_{k}$ be a term that solves a set of dual equations $\mathcal{E}=\left\langle[x \overline{\mathrm{M}} \doteq W],\left[x \overline{\mathrm{M}^{\prime}} \doteq W^{\prime}\right]\right\rangle$, where $W, W^{\prime}$ is a non-constant pair of columns. If $M^{\prime}=\lambda \boldsymbol{y} \cdot z \widehat{M}_{1} \cdots \widehat{M_{k}}$ is a minimal pruning of $M$ that gives minimal approximation of $W, W^{\prime}$ wrt. $\mathcal{E}$, then

- either there exists $j$ such that $\overline{\mathrm{M}}_{j}, \overline{\mathrm{M}}_{j}$ is a pair of splitting columns for $W, W^{\prime}$,

- or there is an address $\gamma$ in $M$ that points to a constant $f \in$ Const $_{M}$ that is totally head accessible.

Proof. The general idea is that we get rid of unimportant occurrences which can be evaluated. This is the place where the definition of self-independent terms plays crucial role.

\section{Proof of Theorem 3:}

The aforementioned lemma allows to prove the main theorem of the paper. This is done by splitting dual interpolation instances. The way the split is done is controlled by the shape of minimal approximation for the hypothetical solution. Details are in appendix.

\section{Decidability}

We have to provide yet another characterisation of solutions for the higher-order matching problem in the case of self-independent terms. This characterisation relies strongly on some constructions for observables.

Definition 8 (pruned observable)

Let $\mathcal{R}$ be an observable. The observable $\mathcal{R}_{C}$ is called a pruning of $\mathcal{R}$ if it contains all the $C$-prunings of terms from $\mathcal{R}$.

Note that $\mathcal{R} \subseteq \mathcal{R}_{C}$ for each $C$.

Definition 9 (observable for terms)

Let $T$ be a set of terms. $\mathcal{R}^{T}$ is a minimal observable containing $T$. 
Note that if $T \subseteq \mathcal{R}$ then $\mathcal{R}^{T} \subseteq \mathcal{R}$.

Definition 10 (pseudo transferring terms)

Let $\mathcal{R}$ be a finite observable, $n, m \in \mathbb{N}$, and $C$ be a set of constants. A term $M: \tau_{1} \rightarrow \cdots \rightarrow \tau_{p} \rightarrow \iota$ is a pseudo $(n, m, C)$-transferring term for the observable $\mathcal{R}$ iff

1. $M=\lambda y_{1} \ldots y_{p} \cdot M^{\prime}$ and $M^{\prime}$ is a term over constants from $C$ without any occurrence of $y_{i}$,

2. $M=\lambda y_{1} \ldots y_{p} . f M_{1} \cdots M_{k}$, where $f \in C$ has a type $\sigma_{1} \rightarrow \cdots \rightarrow \sigma_{k} \rightarrow \iota$ and $\lambda y_{1} \ldots y_{p} . M_{i}$ are pseudo $\left(n_{i}, m, C_{i}\right)$-transferring terms for the observable $\mathcal{R}$ with $n_{i}<n$ and $C_{i}=C \cup\left\{z_{1}, \ldots, z_{r}\right\}$ where $M_{i}=\lambda z_{1} \ldots z_{r} . M_{i}^{\prime}$ and $M_{i}^{\prime}$ does not begin with $\lambda$.

3. $M=\lambda y_{1} \ldots y_{p} . y_{i} M_{1} \cdots M_{k}\left[a \leftarrow N_{a} \boldsymbol{y}, b \leftarrow N_{b} \boldsymbol{y}, z_{0}:=N_{0} \boldsymbol{y}, \ldots, z_{l}:=N_{l} \boldsymbol{y}\right]$, where

$-\boldsymbol{y}=y_{1}, \ldots, y_{p}$

$-z_{0}, \ldots, z_{l}$ have order less than the order of $y_{i}$;

- $\lambda z_{i_{1}} \ldots z_{i_{k}} . M_{i}$ are pseudo $\left(n_{i}, m_{i}, C \cup\{a, b\}\right)$-transferring terms for the observable $\mathcal{R}_{\{a, b\}}$, where $n_{i}$ is the maximal number of equations in a dual set $\mathcal{E}$ characterising a single equivalence class as in Theorem 2, and $m_{i}$ is the maximal total size of right-hand sides in such an $\mathcal{E}$

- $N_{a}$ and $N_{b}$ are pseudo $\left(n_{a}, m_{a}, C\right)$-transferring and pseudo $\left(n_{b}, m_{b}, C\right)$ transferring respectively for the observable $\mathcal{R}$;

- $T$ is a set of terms such that $\mathcal{R}^{T} \varsubsetneqq \mathcal{R}$;

- for each $j$ the term $N_{j}$ is pseudo $\left(n_{j}, m_{j}, C\right)$-transferring for the observable $\mathcal{R}^{T}$

$-n_{a}, n_{b} \leq n$

$-m_{a}+m_{b} \leq m$ and $m_{a}, m_{b} \neq m$

- $n_{j}$ is the number of equations in a dual set characterising an equivalence class of observational equivalence for $\mathcal{R}^{T}$ and $m_{j}$ is the total size of right-hand sides in the aforementioned dual set.

Sometimes when $(n, m, C)$ are unimportant or clearly seen from the context, we shorten the name to pseudo transferring.

We have the following theorem:

Theorem 4. If a dual interpolation set $\mathcal{E}$ for an observable $\mathcal{R}$ has an $(n, m, C)$ transferring solution $M$ then it has a pseudo $(n, m, C)$-transferring solution, too.

We are now ready to conclude with the following theorem.

Theorem 5. The dual interpolation problem for self-independent terms is decidable.

Proof. The decision procedure consists in enumerating all possible pseudo transferring terms for the type of the head variable in an instance of the dual interpolation problem. This may be done recursively according to the definition of pseudo transferring terms.

As an easy corollary we obtain.

Theorem 6. Higher-order matching for self-independent terms is decidable. 


\section{References}

[Bax76] Lewis D. Baxter. The Complexity of Unification. PhD thesis, University of Waterloo, 1976.

[dG00] Philippe de Groote. Linear Higher-Order Matching Is NP-Complete. In Leo Bachmair, editor, Proceedings of 11th International Conference on Rewriting Techniques and Applications, number 1833 in LNCS, pages 127-140. SpringerVerlag, 2000.

[dMS01] Oege de Moor and Ganesh Sittampalam. Higher-order matching for program transformation. Theoretical Computer Science, 269(1-2):135-162, 2001.

[DW02] Daniel J. Dougherty and Tomasz Wierzbicki. A decidable variant of higher order matching. In Sophie Tison, editor, Proc. Thirteenth Intl. Conf. on Rewriting Techniques and Applications (RTA), pages 340-351, 2002.

[Gol81] Warren D. Goldfarb. The undecidability of the second-order unification problem. Theoretical Computer Science, 3:225-230, 1981.

[Har96] John Harrison. HOL light: a tutorial introduction. In Mandayam Srivas and Albert Camilleri, editors, Proceedings of the First International Conference on Formal Methods in Computer-Aided Design, pages 265-269, 1996.

[Hue76] G. Huet. Résolution d'Équations dans les Langages d'Ordre $1,2, \ldots, \omega . \mathrm{PhD}$ thesis, Université de Paris VII, 1976.

[Loa] Ralph Loader. Higher-order $\beta$ matching is undecidable. Can be found at http://homepages. ihug.co.nz/ ${ }^{\sim}$ suckfish/match/.

[Loa01] Ralph Loader. Finitary PCF is not decidable. Theoretical Computer Science, 1-2(266):341-364, 2001.

[MN88] D. Miller and G. Nadathur. An overview of $\lambda$-Prolog. In R. Bowen and K. Kowalski, editors, Proceedings of the Fifth International Logic Programming Conference/ Fifth Symposium on Logic Programming. MIT Press, 1988.

[Pad95a] Vincent Padovani. Decidability of all minimal models. In Stefano Berardi and Mario Coppo, editors, Types for Proofs and Programs, International Workshop TYPES'95, number 1158 in LNCS, pages 201-215. Springer Verlag, 1995.

[Pad95b] Vincent Padovani. On equivalence classes of interpolation equations. In M. Dezani-Ciancaglini and G. Plotkin, editors, Typed Lambda Calculi and Applications, number 902 in LNCS, pages 335-349, 1995.

[Pad96] Vincent Padovani. Filtrage d'Ordre superieur. PhD thesis, Université Paris VII, January 1996.

[Pau89] Lawrence C. Paulson. The foundation of a generic theorem prover. Journal of Automated Reasoning, 5(3):363-397, 1989.

[Sch01] Aleksy Schubert. A note on observational equivalence in the simply typed lambda calculus. Technical Report TR 02-01 (266), Institute of Informatics, Warsaw University, 2001. Can be found at http://www .mimuw .edu.pl/ alx/ftp-public/domains.ps.gz.

[SSS02] Manfred Schmidt-Schauß and Klaus U. Schulz. Decidability of bounded higher-order unification. In CSL 2002, LNCS 2471, pages 522-536. SpringerVerlag, 2002.

[Sta82] Richard Statman. Completness, invariance and $\lambda$-definability. Journal of Symbolic Logic, 1(47), 1982.

[Wol89] David A. Wolfram. The Clausal Theory of Types. PhD thesis, University of Cambridge, 1989. 


\section{A Proofs}

The proofs presented here are only to support the validity of the presented material. They will not be submitted as a part of the paper.

The following lemma allows us to state that absence of unimportant variables guarantees that we are able to construct a splitting column.

Lemma 2. Let $M=\lambda \boldsymbol{y} . z M_{1} \cdots M_{k}$ be a term such that $M$ solves a dual set $\mathcal{E}=$ $\left\langle[x \overline{\mathrm{M}} \doteq W],\left[x \overline{\mathrm{M}^{\prime}} \doteq W^{\prime}\right]\right\rangle$, where $W, W^{\prime}$ is a non-constant pair of columns. If $M^{\prime}=$ $\lambda \boldsymbol{y} . z \widehat{M}_{1} \cdots \widehat{M_{k}}$ is a minimal pruning of $M$ that gives a minimal approximation of $W, W^{\prime}$ wrt. $\mathcal{E}$ and $M^{\prime}$ does not contain unimportant occurrences of any variable from $\boldsymbol{y}$, then terms $\widehat{M}_{i}$ do not contain occurrences of variables from $\boldsymbol{y}$.

Proof. Existence of such a variable means that 0 is unimportant. Details follow.

Suppose, contrary to our claim, that $\gamma=p \cdot \gamma^{\prime}$ is an address in $M^{\prime}$ of a term that starts with a variable $y_{j} \in \boldsymbol{y}$. W.l.o.g. we may assume that $\gamma$ is a $\preceq$-minimal address of this kind. As this address is not unimportant,

1. either $\gamma$ is not totally head accessible,

2. or the term pointed out by $\gamma$ is totally head accessible and there is no $\gamma \cdot \gamma^{\prime}$ totally head accessible.

Case (1). We replace the term at $\gamma$ with $a, b$ or one of constants from $\mathbb{N}$ so that the constraints on pruning and approximation are fulfilled. We obtain this way either an approximation which is less than the original one or a pruning which is less than $M^{\prime}$. This contradicts our assumption that $M^{\prime}$ is a minimal pruning of $M$ that gives a minimal approximation.

Case (2). In this case 0 is an unimportant occurrence. Details are left for the reader.

\section{Proof of Lemma 1:}

The proof is by induction on the number of variables in $M^{\prime}$ that do not play an essential role in constructing the minimal approximation of $W, W^{\prime}$. These variables do not serve as places where a decision is made or places where some symbols are arranged for right-hand sides of equations. They may only produce some terms that are used later on. We can simulate this behaviour by replacing a corresponding variable with an appropriately modified solution of $\mathcal{E}$. This solution contains complete information about the term that will be used later on. A more detailed presentation goes hereafter.

The proof is by induction on the number $p$ of unimportant occurrences of variables from $\boldsymbol{y}$ in $M^{\prime}$.

Case $p=0$. Lemma 2 implies that the head symbol $z$ is either a constant $f \in$ Const $_{M}$ or a variable $y_{j} \in \boldsymbol{y}$. The last case implies immediately that $\overline{\mathrm{M}}^{j}$ is a splitting column with arguments $\widehat{M}_{1}, \ldots, \widehat{M_{k}}$.

Case $p>0$. Let $k \cdot \gamma \cdot 0$ be a maximal address of an unimportant variable $z \in \boldsymbol{y} \boldsymbol{x}$ in $M^{\prime}(\boldsymbol{x}$ are declared on $k \cdot \gamma)$. We have

$$
\gamma\left(\lambda \boldsymbol{y} \cdot \widehat{M}_{k}\right)=z M_{1}^{\prime} \cdots M_{r}^{\prime}\left[\delta \leftarrow N_{\delta} \boldsymbol{y} \boldsymbol{x} \boldsymbol{x}_{\boldsymbol{\delta}}\right]
$$

where 
- $M_{i}^{\prime}=\lambda z_{1} \ldots z_{r_{i}} \cdot \widetilde{M}_{i}$ are closed terms over constants Const $_{i}$, and $\widetilde{M}_{i}$ does not begin with $\lambda$;

$-\varepsilon\left(N_{\delta}\right)$ begins with a symbol from $\boldsymbol{y} \boldsymbol{x}$ or with a constant, and $\delta$ is totally head accessible;

- $\boldsymbol{x}_{\boldsymbol{\delta}}$ are declared in $z M_{1}^{\prime} \cdots M_{r}^{\prime}$ on the path between $\gamma$ and $\delta$.

We have several cases according to the form of $N_{\delta}$.

1. The head symbol of $N_{\delta}$ is a constant. The form of (2) implies that $M$ can be presented as

$$
M=C_{M}\left[z \overline{M_{1}^{\prime}} \cdots \overline{M_{r}^{\prime}}\left[\delta \leftarrow \overline{N_{\delta}} \boldsymbol{y} \boldsymbol{x} \boldsymbol{x}_{\boldsymbol{\delta}}\right]\right]
$$

where $M_{1}^{\prime}, \ldots, M_{r}^{\prime}, N_{\delta}$ are corresponding prunings of $\overline{M_{1}^{\prime}}, \ldots, \overline{M_{r}^{\prime}}, \overline{N_{\delta}}$ and $C_{M}[\bullet]$ is a suitable context. The term $\overline{N_{\delta}}$ begins with a constant. This term cannot be a constant term (with no occurrences of variables from $\boldsymbol{y} \boldsymbol{x}_{\boldsymbol{\delta}}$ ), since $W, W^{\prime}$ is not a constant pair. This means that $\overline{N_{\delta}}$ begins with a function symbol. Similarly, the function symbol starts $N_{\delta}$. This means that the address $\delta \cdot 0$ is a path to a totally head accessible constant function symbol.

2. The head symbol of $N_{\delta}$ is $x \in \boldsymbol{y} \boldsymbol{x}$. We define a context $C_{\gamma}[\bullet]$ as follows: $C_{\gamma}[\gamma(M)]=M$. The term $M_{\text {new }}$ is defined as

$$
M_{\text {new }}=C_{\gamma}\left[\overline{N_{\delta}} \boldsymbol{y} \boldsymbol{x} R_{1} \cdots R_{p}\right] .
$$

Let $x_{i} \in \boldsymbol{x}_{\boldsymbol{\delta}}$ (with $x_{i}: \tau_{1} \rightarrow \cdots \rightarrow \tau_{r} \rightarrow \iota$ ), we define

$$
R_{i}=\lambda z_{1} \ldots z_{r} . C_{\gamma}\left[z M_{1}^{\prime} \cdots M_{r}^{\prime}\left[c \leftarrow x_{i} z_{1} \cdots z_{r}\right]\right] \boldsymbol{y} .
$$

It is easily verified that $M_{\text {new }}$ is a solution of $\mathcal{E}$. It is so because $M$ is selfindependent. Let

$$
M_{\text {new }}^{\prime}=C_{\gamma}\left[N_{\delta} \boldsymbol{y} \boldsymbol{x}\left(\lambda \boldsymbol{z}_{1} \cdot c_{1}\right) \cdots\left(\lambda \boldsymbol{z}_{1} \cdot c_{p}\right)\right]
$$

with $c_{1}, \ldots, c_{p} \in\{a, b\} \cup \mathbb{N}$ and the constants $c_{1}, \ldots, c_{p}$ inserted so that conditions for pruning and approximation are met. Note that $M_{\text {new }}^{\prime}$ is a pruning of $M_{\text {new }}$.

It is not possible that only one constant in $\left\{c_{1}, \ldots, c_{p}\right\}$ is totally head accessible, since otherwise $\gamma$ is not the maximal occurrence of an unimportant symbol.

The term $M_{\text {new }}^{\prime}$ is a non-trivial pruning. Triviality of $M_{\text {new }}^{\prime}$ implies either triviality of $M^{\prime}$ or that $k \cdot \gamma$ is not a maximal address of an unimportant variable.

As $M_{\text {new }}^{\prime}$ is a non-trivial pruning, we have a minimal non-trivial pruning $M_{\text {new }}^{\prime \prime}$ of $M_{\text {new }}$ wrt. $W, W^{\prime}$. The term $M_{\text {new }}^{\prime \prime}$ has strictly less occurrences of unimportant variables than $M^{\prime}$. Thus, we can apply the induction hypothesis and obtain that either some $\overline{\mathrm{M}}^{j}$ is a splitting column or that there exists an address $\gamma^{\prime}$ in $M_{\text {new }}^{\prime \prime}$ which is an occurrence of a totally head accessible constant symbol $f$. If $\gamma^{\prime}=k \cdot \gamma \cdot \widehat{\gamma} \cdot 0$ then we obtain an address $\gamma^{\prime \prime}$ of $f$ in $M^{\prime}$ as $\gamma^{\prime \prime}=k \cdot \gamma \cdot \gamma_{c} \cdot \widehat{\gamma} \cdot 0$ where $\gamma_{c}\left(z M_{1}^{\prime} \cdots M_{r}^{\prime}\right)=c$. If $\gamma^{\prime} \neq k \cdot \gamma \cdot \widehat{\gamma} \cdot 0$ then this $\gamma^{\prime}$ is also an occurrence of $f$ in $M^{\prime}$. 


\section{Proof of Theorem 3:}

Let $\mathcal{E}=\left\langle E, E^{\prime}\right\rangle=\left\langle[x \overline{\mathrm{M}} \doteq W],\left[x \overline{\mathrm{M}^{\prime}} \doteq W^{\prime}\right]\right\rangle$. We define the value $n$ as the number of symbols in $W, W^{\prime}$, the value $m$ as the number of equations in $\mathcal{E}$, the value $h$ as the height of $M$, and the set $C$ as the set of constants in $\mathcal{E}$.

The proof is by induction wrt. lexicographic order on triples $\langle n, m, h\rangle$.

If $W, W^{\prime}$ is a constant pair of columns, then $M^{\prime}=\lambda \boldsymbol{y} \cdot W^{1}$ is a transferring solution.

If $m=1$, then $M^{\prime}=\lambda \boldsymbol{y} \cdot W^{1}$ is a transferring solution provided that $E \neq \emptyset$. If $E=\emptyset$ then $M^{\prime}=\lambda \boldsymbol{y} \cdot \operatorname{NFL}\left(M{\overline{\mathrm{M}^{\prime}}}_{1}^{1} \cdots{\overline{\mathrm{M}^{\prime}}}_{m}^{1}\right)$ is a transferring solution.

If $h=1$, then $M=\lambda \boldsymbol{y}$.c for some constant $c$ of a base type or $c \in \boldsymbol{y}$. In this case, $M^{\prime}=M$.

If $n=1$, then this reduces to the case when $m=1$.

If $\langle n, m, h\rangle>\langle 1,1,1\rangle$, then we have two subcases

1. $M=\lambda \boldsymbol{y} \cdot f M_{1} \cdots M_{k}$ where $f \in C$;

2. $M=\lambda \boldsymbol{y} \cdot y_{i} M_{1} \cdots M_{k}$ for some $y_{i} \in \boldsymbol{y}$.

Case $M=\lambda \boldsymbol{y} . f M_{1} \cdots M_{k}$. Let $M_{i}=\lambda z_{1}^{i} \ldots z_{r_{i}}^{i} \cdot \widetilde{M}_{i}$ where $\widetilde{M}_{i}$ does not begin with $\lambda$. In this case, all terms in $W$ begin with $f$ :

$$
W=\left\langle f B_{1}^{1} \cdots B_{k}^{1}, \ldots, f B_{1}^{p} \cdots B_{k}^{p}\right\rangle
$$

and some terms of $W^{\prime}$, say in sub-column $W^{\prime \prime}$ :

$$
W^{\prime \prime}=\left\langle f B_{1}^{\prime \prime 1} \cdots B_{k}^{\prime \prime 1}, \ldots, f B_{1}^{\prime \prime p^{\prime}} \cdots B_{k}^{\prime \prime p^{\prime}}\right\rangle .
$$

We define columns $W_{1}, \ldots, W_{k}$ and $W_{1}^{\prime}, \ldots, W_{k}^{\prime}$ as $W_{i}=\left\langle B_{i}^{1}, \ldots, B_{i}^{p}\right\rangle$, and $W_{i}^{\prime}=$ $\left\langle B_{i}^{\prime \prime 1}, \ldots, B_{i}^{\prime \prime \prime}\right\rangle$. We can define sets of equations $E_{i}=\left[x \overline{\mathrm{M}} \doteq W_{i}\right]$ and $E_{i}^{\prime}=$ $\left[x \overline{\mathrm{M}^{\prime \prime}} \doteq W_{i}^{\prime}\right]\left(\overline{\mathrm{M}^{\prime \prime}}\right.$ is a sub-matrix of $\overline{\mathrm{M}^{\prime}}$ corresponding to $\left.W^{\prime \prime}\right)$. It is easily verified that $\left\langle E_{i}, E_{i}^{\prime}\right\rangle$ has a solution $\lambda \boldsymbol{y} \cdot \widetilde{M}_{i}$. Let the value $n_{i}$ be the number of symbols in $W_{i} W_{i}^{\prime}$, the value $m_{i}$ be the number of equations in $E_{i} \cup E_{i}^{\prime}$ and $C_{i}=C \cup$ $\left\{z_{1}^{i}, \ldots, z_{r_{i}}^{i}\right\}$. The number $n_{i}$ is strictly less than the number of symbols $n$ so by the induction hypothesis we have $\left(n_{i}, m_{i}, C_{i}\right)$-transferring solutions $\lambda \boldsymbol{y} . \widetilde{M}_{i}^{\prime}$ of sets $\left\langle E_{i}, E_{i}^{\prime}\right\rangle$. Thus, $\lambda \boldsymbol{y} . f M_{1}^{\prime} \cdots M_{k}^{\prime}$ is a $(n, m, C)$-transferring solution of $\mathcal{E}$ where $M_{i}^{\prime}=\lambda z_{1}^{i} \ldots z_{r_{i}}^{i} \cdot \widetilde{M_{i}^{\prime}}$.

Case $M=\lambda \boldsymbol{y} \cdot y_{i} M_{1} \cdots M_{k}$ for some $y_{i} \in \boldsymbol{y}$.

The pair of columns $W, W^{\prime}$ has a minimal approximation. Let $M^{\prime}$ be a minimal pruning for the minimal approximation of $W, W^{\prime}$. Lemma 1 implies that either $\overline{\mathrm{M}}_{j}, \overline{\mathrm{M}}_{j}$ are splitting columns for $W, W^{\prime}$ or there exists a totally head accessible occurrence in $M$ of a constant $f \in$ Const $_{M}$.

Case when certain $\overline{\mathrm{M}}_{j}, \overline{\mathrm{M}}_{j}^{\prime}$ are splitting columns for $W, W^{\prime}$. The definition of splitting columns says that there exist terms $P_{1}, \ldots, P_{r}$ such that $\mathrm{NFL}\left(\overline{\mathrm{M}}_{j} P_{1} \cdots P_{r}\right)=$ $\widetilde{W}$ and $\operatorname{NFL}\left(\overline{\mathrm{M}}_{j}^{\prime} P_{1} \cdots P_{r}\right)=\widetilde{W^{\prime}}$ where $\widetilde{W}, \widetilde{W^{\prime}}$ is an approximation of $W, W^{\prime}$. We produce on the basis of $\mathcal{E}$ new dual sets of equations $\mathcal{E}_{a}, \mathcal{E}_{b}, \mathcal{E}_{0}, \ldots, \mathcal{E}_{l}$. These sets are defined as $\mathcal{E}_{c}=\left\langle E_{c}, E_{c}^{\prime}\right\rangle$ where 
1. $E_{a}=\left\{\left[x \overline{\mathrm{M}}_{1}^{k} \cdots \overline{\mathrm{M}}_{m}^{k}=W^{k}\right] \mid \operatorname{NFL}\left(\overline{\mathrm{M}}_{j}^{k} P_{1}, \ldots, P_{r}\right)=a\right\}$, and $E_{a}^{\prime}=\left\{\left[x{\overline{\mathrm{M}^{\prime}}}_{1}^{k} \ldots{\overline{\mathrm{M}^{\prime}}}_{m}^{k}=W^{\prime k}\right] \mid \operatorname{NFL}\left({\overline{\mathrm{M}^{\prime}}}_{j}^{k} P_{1}, \ldots, P_{r}\right)=a\right\}$,

2. $E_{b}=\left\{\left[x \overline{\mathrm{M}}_{1}^{k} \ldots \overline{\mathrm{M}}_{m}^{k}=W^{k}\right] \mid \operatorname{NFL}\left(\overline{\mathrm{M}}_{j}^{k} P_{1}, \ldots, P_{r}\right)=b\right\}$, and $E_{b}^{\prime}=\left\{\left[x \overline{\mathrm{M}^{\prime}}{ }_{1}^{k} \ldots \overline{\mathrm{M}^{\prime}}{ }_{m}^{k}=W^{\prime k}\right] \mid \operatorname{NFL}\left({\overline{\mathrm{M}^{\prime}}}_{j}^{k} P_{1}, \ldots, P_{r}\right)=b\right\}$,

3 . the definition of $E_{i}, E_{i}^{\prime}$ for $i \in \mathbb{N}$ is more complex and presented hereafter.

Let $[x \overline{\mathrm{N}} \doteq \widehat{W}]=E \backslash\left(E_{a} \cup E_{b}\right)$ and $\left[x \overline{\mathrm{N}^{\prime}} \doteq \widehat{=} \widehat{W}^{\prime}\right]=E^{\prime} \backslash\left(E_{a}^{\prime} \cup E_{b}^{\prime}\right)$. We have

$$
\begin{aligned}
& \operatorname{NFL}\left(\overline{\mathrm{M}}_{j}^{k} P_{1} \cdots P_{r}\right)=\widehat{W}^{k}=C^{k}\left[n_{1}^{k}, \ldots, n_{q}^{k}\right] \\
& \operatorname{NFL}\left({\overline{\mathrm{M}^{\prime}}}_{j}^{k} P_{1} \cdots P_{r}\right)=\widehat{W}^{\prime k}=C^{\prime k}\left[n_{1}^{k}, \ldots, n_{q^{\prime}}^{k}\right]
\end{aligned}
$$

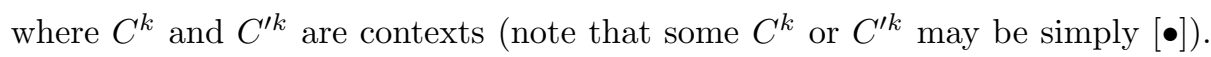
Define

$$
\begin{aligned}
& \Pi^{k}\left(n_{s}^{k}\right)=\left\{\gamma \mid \gamma \text { points out on a constant } n_{s}^{k} \text { in } \widehat{W}^{k}\right\}, \\
& \Pi^{\prime k}\left(n_{s}^{k}\right)=\left\{\gamma \mid \gamma \text { points out on a constant } n_{s}^{k} \text { in } \widehat{W}^{\prime}\right\}
\end{aligned}
$$

We establish sets of equations

$$
\begin{aligned}
E_{n}=\left\{\left[x^{n} \overline{\mathrm{N}}_{1}^{k} \cdots \overline{\mathrm{N}}_{m}^{k} O_{1}^{k} \cdots O_{l^{\prime}}^{k} \doteq \gamma\left(W^{k}\right)\right]\right. & \left.\mid \gamma \in \Pi^{k}(n) \text { and } k \in \mathbb{N}\right\} \\
E_{n}^{\prime}=\left\{\left[x^{n}{\overline{\mathrm{N}^{\prime}}}_{1}^{k} \cdots{\overline{\overline{N^{\prime}}}}_{m}^{k} O_{1}^{\prime k} \cdots O_{l^{\prime}}^{\prime k} \doteq \gamma\left(W^{\prime k}\right)\right] \mid\right. & \gamma \in \Pi^{k}(n) \text { and } k \in \mathbb{N} \\
& \text { and } \left.\widetilde{W^{\prime}}{ }^{k} \text { meets with } W^{\prime k}\right\}
\end{aligned}
$$

where terms $O_{k^{\prime}}^{k}$ for $k^{\prime} \in\left\{1, \ldots, l^{\prime}\right\}$ are defined as follows. Let $\gamma_{k}$ be an occurrence of a constant $n_{s}^{k}$ in $M^{\prime}$ that results in $n_{s}^{k}$ in $\widehat{W}^{k}\left(\right.$ or $\left.\widehat{W}^{\prime k}\right)$. Let

$$
M^{\prime \prime}=M\left[\gamma_{k} \leftarrow Z x_{1} \cdots x_{l^{\prime}}\right]
$$

where $Z$ is a fresh constant of a suitable type and $\left\{x_{1}, \ldots, x_{l^{\prime}}\right\}=\mathrm{FV}\left(\gamma_{k}(M)\right) \backslash \boldsymbol{y}$. We have

$$
\begin{aligned}
& \operatorname{NFL}\left(M^{\prime \prime} \overline{\mathrm{N}}_{1}^{k} \ldots \overline{\mathrm{N}}_{m}^{k}\right)=C^{k}\left[B_{1}, \ldots,\left(Z \bar{O}_{1}^{k} \ldots \bar{O}_{l^{\prime}}^{k}\right), \ldots, B_{q}\right] \\
& \operatorname{NFL}\left(M^{\prime \prime}{\overline{\mathrm{N}^{\prime}}}_{1}^{k} \ldots{\overline{\mathrm{N}^{\prime}}}_{m}^{k}\right)=C^{\prime k}\left[B_{1}^{\prime} \ldots,\left(Z \bar{O}_{1}^{\prime k} \ldots \bar{O}_{l^{\prime}}^{\prime k}\right), \ldots, B_{q^{\prime}}^{\prime}\right],
\end{aligned}
$$

We can also mimic reduction steps as above in

$$
\begin{aligned}
& \operatorname{NFL}\left(M \overline{\mathrm{N}}_{1}^{k} \ldots \overline{\mathrm{N}}_{m}^{k}\right) \rightarrow{ }_{\beta \eta}^{*} C^{k}\left[B_{1}, \ldots,\left(M^{\#} \widehat{O}_{1}^{k} \ldots \widehat{O}_{l^{\prime}}^{k}\right), \ldots, B_{q}\right], \\
& \operatorname{NFL}\left(M{\overline{\mathrm{N}^{\prime}}}_{1}^{k} \ldots{\overline{\mathrm{N}^{\prime}}}_{m}^{k}\right) \rightarrow_{\beta \eta}^{*} C^{\prime k}\left[B_{1}^{\prime} \ldots,\left(M^{\#} \widehat{O}_{1}^{\prime k} \ldots \widehat{O}_{l^{\prime}}^{\prime k}\right), \ldots, B_{q^{\prime}}^{\prime}\right] .
\end{aligned}
$$

At last we define $O_{k^{\prime}}^{k}$ (and $\left.O_{k^{\prime}}^{\prime k}\right)$ as

$$
\begin{aligned}
& O_{k^{\prime}}^{k}=\bar{O}_{k^{\prime}}^{k}\left[Z:=M^{\#}\right], \\
& O_{k^{\prime}}^{\prime k}=\bar{O}_{k^{\prime}}^{\prime k}\left[Z:=M^{\#}\right] .
\end{aligned}
$$

The dual sets $\mathcal{E}_{a}, \mathcal{E}_{b}, \mathcal{E}_{0}, \ldots, \mathcal{E}_{s}$ are solvable by the terms $M, M, \widetilde{M}_{0}, \ldots \widetilde{M}_{s}$ respectively. We define $\widetilde{M}_{i}$ as

$$
\lambda \boldsymbol{y} x_{1} \ldots x_{l^{\prime}} \cdot \gamma_{k}(M) .
$$


The sets $\mathcal{E}_{a}, \mathcal{E}_{b}$ have both the number of equations and the number of symbols occurrences less than $\mathcal{E}$. In the sets $\mathcal{E}_{0}, \ldots, \mathcal{E}_{l}$, the size of right-hand sides is less than the size of $W W^{\prime}$ (constants from $\mathbb{N}$ are guarded). This means that we can apply the induction hypothesis and obtain transferring solutions $\dot{M}_{a}, \dot{M}_{b}, \dot{M}_{0}, \ldots \dot{M}_{l}$ of $\mathcal{E}_{a}, \mathcal{E}_{b}, \mathcal{E}_{0}, \ldots, \mathcal{E}_{l}$. We define a term

$$
\begin{aligned}
\dot{M}=\lambda \boldsymbol{y} \cdot y_{j} P_{1} \cdots P_{r}[ & a \leftarrow \dot{M}_{a} \boldsymbol{y}, \\
& b \leftarrow \dot{M}_{b} \boldsymbol{y}, \\
& 0 \leftarrow \dot{M}_{0} \boldsymbol{y} \boldsymbol{x}_{\mathbf{0}}, \\
& \ldots, \\
& \left.l \leftarrow \dot{M}_{l} \boldsymbol{y} \boldsymbol{x}_{\boldsymbol{l}}\right]
\end{aligned}
$$

where $\boldsymbol{x}_{\boldsymbol{i}}$ are variables bound in the $P_{k}$ in which $i$ occurs as a constant.

The term $\dot{M}$ is a solution, because when we reduce it with left-most, outmost strategy applied in equations of $\mathcal{E}$, then we reach either $\dot{M}_{a} \overline{\mathrm{M}}^{k}, \dot{M}_{b} \overline{\mathrm{M}}^{k}$ or $C^{k}\left[\left(\dot{M}_{n_{1}^{k}} \cdots\right) \cdots\left(\dot{M}_{n_{q}^{k}} \cdots\right)\right]$. As $\dot{M}_{a}, \dot{M}_{b}, \dot{M}_{0}, \ldots \dot{M}_{l}$ are solutions of equations $\mathcal{E}_{a}, \mathcal{E}_{b}, \mathcal{E}_{0}, \ldots, \mathcal{E}_{l}$, we immediately see that reached terms reduce further to appropriate subterms of right-hand sides $W, W^{\prime}$. Note that so defined $\dot{M}$ is $(n, m, C)$ transferring solution of $\mathcal{E}$.

Case when there exists an occurrence of a constant $f$ in $M$ which is totally head accessible. This case is technically similar to the case when $M=$ $\lambda \boldsymbol{y} . f M_{1} \cdots M_{k}$ The only difficulty concerns free variables that occur in $\gamma(M)$ where $\gamma$ is the intervening occurrence of $f$ in $M$. We can get rid of them using the trick employed in (4). Details are left for the reader.

\section{Proof of Theorem 4:}

Induction on lexicographically ordered pairs $\langle o, h, m\rangle$ where $o$ is the order of the type of $M, h$ is the height of $M$ and $m$ is the number of terms in $\mathcal{R}$.

If $o=0$ then $\mathcal{E}=\left\langle E, E^{\prime}\right\rangle$ may have only one equation in $E$ and the equation has the form $x \doteq M$. In this case, $M$ is pseudo transferring.

If $h=1$ then $M=\lambda \boldsymbol{y} . c$ where $c$ is a base type constant. In this case, $M$ is pseudo transferring, too.

If $m=1$ then $\mathcal{R}$ is a single term, so there can only be one equation characterising $M$ and thus $M$ is pseudo transferring.

Let $\langle o, h, m\rangle>\langle 0,1,1\rangle$. We have three subcases here according to the transferring structure of $M$. The only non-trivial case is when $M$ has the form (3). As $N_{a}, N_{b}$ have depth less then $M$, we have by the induction hypothesis pseudo transferring terms $\overline{N_{a}}, \overline{N_{b}}$ with $n_{a}, m_{a}, n_{b}, m_{b}$ as in $M$.

Let $M_{i}^{\prime}=M_{i}\left[i_{1} \leftarrow z_{i_{1}} \boldsymbol{x}_{\boldsymbol{i}_{1}}, \ldots, i_{k} \leftarrow z_{i_{k}} \boldsymbol{x}_{\boldsymbol{i}_{\boldsymbol{k}}}\right]$ where $\left\{i_{1}, \ldots, i_{k}\right\}$ are constants from $\mathbb{N}$ that occur in $M_{i}$. By Theorem 2 and Theorem 3, we can generate transferring equivalents $\lambda z_{1} \cdot \widehat{M_{1}}, \ldots, \lambda z_{\boldsymbol{k}} \widehat{M_{k}}$ of corresponding terms $\lambda \boldsymbol{z}_{1} \cdot M_{1}^{\prime}, \ldots, \lambda \boldsymbol{z}_{1} \cdot M_{k}^{\prime}$. This equivalents are taken for the observable $\mathcal{R}_{\{a, b\}}$. This ensures that terms 
$\mathrm{M}_{i} \widehat{M_{1}} \cdots \widehat{M_{k}}$ give the same approximations as $\mathrm{M}_{i} M_{1} \cdots M_{k}$ and terms

$$
\begin{aligned}
\mathrm{M}_{i} \widehat{M_{1}} \cdots \widehat{M_{k}}[ & a \leftarrow N_{a} \boldsymbol{y}, \\
& b \leftarrow N_{b} \boldsymbol{y}, \\
& z_{0}:=N_{0} \boldsymbol{y}, \\
& \cdots, \\
& \left.z_{l}:=N_{l} \boldsymbol{y}\right]
\end{aligned}
$$

give the same results as

$$
\begin{aligned}
\mathrm{M}_{i} M_{1} \cdots M_{k}[ & a \leftarrow N_{a} \boldsymbol{y}, \\
b & \leftarrow N_{b} \boldsymbol{y}, \\
0 \leftarrow N_{0} \boldsymbol{y} \boldsymbol{x}, & \\
& \cdots, \\
& \left.l \leftarrow N_{l} \boldsymbol{y} \boldsymbol{x}\right] .
\end{aligned}
$$

As terms $\lambda z_{1} \cdot \widehat{M}_{1}, \ldots, \lambda z_{k} \widehat{M_{k}}$ have order less than $o$, we can obtain by the induction hypothesis terms $\lambda \boldsymbol{z}_{1} \cdot \overline{M_{1}}, \ldots, \lambda \boldsymbol{z}_{\boldsymbol{k}} \overline{M_{k}}$ which are pseudo $\left(n^{\prime}, m^{\prime}, C \cup \mathrm{Const}_{i}\right)$ transferring with $\left(n^{\prime}, m^{\prime}, C \cup\right.$ Const $\left._{i}\right)$ as in the corresponding $\widehat{M}_{i}$ terms.

The last step is to obtain pseudo transferring equivalents of $N_{0}, \ldots, N_{l}$. This can be done as these terms are used in

$$
\begin{aligned}
\mathrm{M}_{i} \widehat{M_{1}} \cdots \widehat{M_{k}}[ & a \leftarrow N_{a} \boldsymbol{y}, \\
& b \leftarrow N_{a} \boldsymbol{y}, \\
& z_{0}:=N_{0} \boldsymbol{y}, \\
& \cdots, \\
& \left.z_{l}:=N_{l} \boldsymbol{y}\right]
\end{aligned}
$$

only in contexts resulting in terms from $\mathcal{R}^{T}$ where $T$ is the set of right-hand sides of equations defined as in (3) in the proof of Theorem 3. As $\mathcal{R}^{T} \subset \mathcal{R}$, we obtain by the induction hypothesis $\mathcal{R}^{T}$ equivalents $\overline{N_{0}}, \ldots, \overline{N_{l}}$ of terms $N_{0}, \ldots, N_{l}$.

Now, we can conclude with the construction of a pseudo $(n, m, C)$-transferring term

$$
\begin{aligned}
\lambda y_{1} \ldots y_{p} \cdot y_{i} \overline{M_{1}} \cdots \overline{M_{k}}[ & a \leftarrow \overline{N_{a}} \boldsymbol{y}, \\
& b \leftarrow \overline{N_{a}} \boldsymbol{y}, \\
& z_{0}:=\overline{N_{0}} \boldsymbol{y}, \\
& \cdots, \\
& \left.z_{l}:=\overline{N_{l}} \boldsymbol{y}\right] .
\end{aligned}
$$

\section{Proof of Theorem 5:}

The decision procedure consists in enumerating all possible pseudo transferring terms for the type of the head variable in an instance of the dual interpolation problem. This may be done recursively according to the definition of pseudo transferring terms. The recursion is parametrised with

- the type order $o$ of head variable, 
- the number of terms $r$ in an observable,

- the number of symbols $n$ in right-hand sides of the given instance of the dual interpolation problem,

- the number of equations $m$ in the instance.

These numbers constitute a lexicographic measure for the recursion.

In the case (1) of Definition 10, the algorithm stops.

In the case (2) of Definition 10, $n$ decreases while other parameters stay intact.

In the case (3) of Definition 10, we have the following subcases.

- For $N_{a}$ and $N_{b}$ the number $m$ decreases while $n$ does not increase and other parameters stay intact.

- For $N_{0}, \ldots, N_{l}$ the number of terms in observable decreases since $\mathcal{R}^{T} \subset \mathcal{R}$. The order stays intact.

- For $M_{1}, \ldots, M_{l}$ the order decreases.

The correctness is based on the knowledge that solutions for the dual interpolation problems are representants for all equivalence classes in the observational equivalence $\approx_{\mathcal{R}}$ where $\mathcal{R}$ is an observable (see Theorem 2 ). 